\title{
High throughput microRNAs sequencing profile of serum exosomes in women with and without polycystic ovarian syndrome
}

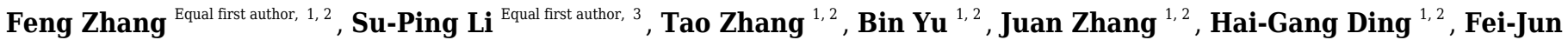 \\ Ye $^{4}$, Hua Yuan ${ }^{1,2}$, Ying-Ying Ma ${ }^{1,2}$, Hai-Tao Pan ${ }^{\text {Corresp., 1, } 2, \text { Yao He }}{ }^{\text {Corresp. 1, } 2}$ \\ ${ }^{1}$ Shaoxing Maternity and Child Health Care Hospital, Shaoxing, China \\ 2 Obstetrics and Gynecology Hospital of Shaoxing University, Shaoxing, China \\ 3 Jiaxing University Affiliated Women and Children's Hospital, Jiaxing, China \\ 4 Zhoushan Maternity and Child Health Care Hospital, Zhoushan, China \\ Corresponding Authors: Hai-Tao Pan, Yao He \\ Email address: panhaitao2007@163.com, sxfbheyao@163.com
}

Background: Polycystic ovary syndrome (PCOS) is the most common type of endocrine disorder, affecting $5-11 \%$ of women of reproductive age worldwide. microRNAs (miRNAs) stably exist in circulating blood encapsulated in extracellular vesicles such as exosomes; therefore, serum miRNAs have the potential to serve as novel PCOS biomarkers.

Methods: To identify miRNA biomarkers that are associated with PCOS, we performed a comprehensive sequence-based characterization of the PCOS serum miRNA landscape. The serum exosomes were successfully isolated and characterized in a variety of ways. Next, sequence-based analysis was performed on serum exosomes to screen the differentially expressed miRNAs in women with and without PCOS.

Results: The sequence data revealed that the levels of 54 miRNAs significantly differed between PCOS patients and normal controls. The levels of these miRNAs were detected by RT-qPCR. The results show that hsa-miR-1299, hsa-miR-6818-5p hsa-miR-192-5p, and hsa-miR-145-5p are significantly differentially expressed in PCOS patients serum exosomes and identify these microRNAs as potential biomarkers for PCOS. Furthermore, Gene Ontology (GO) analyses and KEGG pathway analyses of the miRNA targets further allowed to explore the potential implication of the miRNAs in PCOS.

Conclusion: Our findings suggest that serum exosomal miRNAs serve important roles in PCOS and may be used as novel molecular biomarkers for clinical diagnosis. 


\section{High throughput microRNAs sequencing profile of}

\section{2 serum exosomes in women with and without}

\section{3 polycystic ovarian syndrome}

4

5

6

8 10

27

28

29

30

Feng Zhang ${ }^{1,2 *}$, Su-Ping Li $^{3, *}$, Tao Zhang ${ }^{1,2}$, Bin $\mathrm{Yu}^{1,2}$, Juan Zhang ${ }^{1,2}$, Hai-Gang Ding ${ }^{1,2}$, Fei-Jun $\mathrm{Ye}^{3}$, Hua Yuan ${ }^{1,2}$, Ying-Ying Ma ${ }^{1,2}$, Hai-Tao $\operatorname{Pan}^{1,2}$, Yao $\mathrm{He}^{1,2} \varpi$

${ }^{1}$ Shaoxing Maternity and Child Health Care Hospital, Shaoxing, China.

${ }^{2}$ Obstetrics and Gynecology Hospital of Shaoxing University, Shaoxing, China

${ }^{3}$ Jiaxing University Affiliated Women and Children's Hospital, Jiaxing, China

${ }^{4}$ Zhoushan Maternity and Child Health Care Hospital, Zhoushan, China.

* Feng Zhang and Su-Ping Li contributed to this study equally.

Corresponding Author:

Yao $\mathrm{He}$

Shaoxing Maternity and Child Health Care Hospital, Shaoxing, 312000, China.

Email address: sxfbheyao@163.com

Or to:

Hai-Tao Pan

Shaoxing Maternity and Child Health Care Hospital, Shaoxing, 312000, China.

E-mail address: panhaitao2007@163.com 


\section{Abstract}

32 Background: Polycystic ovary syndrome (PCOS) is the most common type of endocrine 33 disorder, affecting 5-11\% of women of reproductive age worldwide. microRNAs (miRNAs)

34 stably exist in circulating blood encapsulated in extracellular vesicles such as exosomes;

35 therefore, serum miRNAs have the potential to serve as novel PCOS biomarkers.

36 Methods: To identify miRNA biomarkers that are associated with PCOS, we performed a comprehensive sequence-based characterization of the PCOS serum miRNA landscape. The serum exosomes were successfully isolated and characterized in a variety of ways. Next, sequence-based analysis was performed on serum exosomes to screen the differentially expressed miRNAs in women with and without PCOS.

Results: Our sequencing data revealed that the levels of 54 miRNAs significantly differed between PCOS patients and normal controls. The levels of these miRNAs were detected by RTqPCR. The results show that hsa-miR-1299, hsa-miR-6818-5p, hsa-miR-192-5p, and hsa-miR$145-5 p$ are differentially expressed in PCOS patients serum exosomes and identify these microRNAs as potential biomarkers for PCOS. Furthermore, Gene Ontology (GO) analyses and KEGG pathway analyses of the miRNA targets further allowed to explore the potential implication of the miRNAs in PCOS.

Conclusion: Our findings suggest that serum exosomal miRNAs serve important roles in PCOS and may be used as novel molecular biomarkers for clinical diagnosis.

50

\section{Introduction}

Polycystic ovary syndrome (PCOS) is the most common infertility disorder in reproductive-aged women. Metabolic issues like inflammation, increased coagulability, visceral obesity, insulin resistance and androgen excess are considered as key features in $\operatorname{PCOS}^{1}$. PCOS is a multifactorial disorder that affects $5-11 \%$ of women of reproductive age worldwide ${ }^{1}$. Exosomes are membrane-bound vesicles that are released into body fluids such as serum, plasma, urine and saliva $^{2}$. In particular, plasma and serum exosome microRNAs (miRNAs) profiling have been shown to have a potential in the diagnosis of different diseases ${ }^{3}$. 
62

63 Exosomes are a class of extracellular vesicles (EVs) that are secreted by most cell types of the

64 body able to transport biologically active cargo between cells, including protein, lipids, mRNA

65 and miRNA $^{2}$. microRNAs (miRNAs) are a class of endogenous and highly conserved non-

66 coding small RNA, with the length from 18 to 24 nucleotides. It has been previously reported

67 that the small RNA sequences contained in human follicular fluid (HFF) exosomes may play a

68 key role in the mechanism that drives PCOS pathogenesis, and thereby can act as molecular

69 biomarkers for PCOS diagnosis in the future ${ }^{4}$. Mesenchymal stem cells derived exosomal miR-

$70 \quad 323-3 p$ promotes proliferation and inhibit apoptosis of cumulus cells in polycystic ovary syndrome (PCOS) ${ }^{5}$. PCOS serum-derived exosomal miR-27a-5p stimulates endometrial cancer cells migration and invasion, indicating that serum exosomal miR-27a-5p may play a role in EC development in PCOS patients ${ }^{6}$. circRNAs profile of follicle fluid exosomes has also been sequenced in polycystic ovary syndrome patients ${ }^{7}$. In addition, S100-A9 protein in exosomes derived from follicular fluid promotes inflammation via activation of NF-kappaB pathway in

76 polycystic ovary syndrome ${ }^{8}$. Although other studies have reported alterations in miRNA in

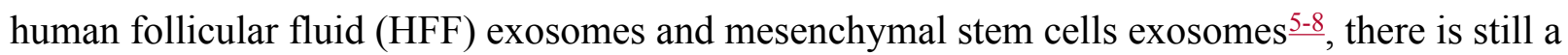
need for further research in promising therapeutic and prognostic serum biomarkers in PCOS.

Based on previous studies, it is likely that serum exosomes and their contents, particularly miRNAs, may serve as potential biomarkers for PCOS. Therefore, this study aimed to investigate and compare the expression of these miRNAs in serum exosomes of women with and without polycystic ovary syndrome (PCOS). The findings of this study may provide a new biomarker and therapeutic target that facilitates early diagnosis and treatment for PCOS.

\section{Materials \& Methods}

\subsection{Study participants}

8 The present study was approved by the Ethics Committee of Shaoxing Maternity and Child Health Care Hospital (Ethical Application Ref: 2018024). All participants signed an appropriate 0 informed consent form. Serum samples from 4 healthy female donors were collected as normal 1 control, while serum obtained from PCOS according to the Rotterdam standard served as the 2 experimental group $(n=4)$. Their clinical characteristics are outlined in Table 1. 
93

\section{$94 \quad 2.2$ Exosomes purification and characterization}

95 To isolate exosomes, all samples were filtered through $0.22 \mu \mathrm{M}$ filters. Exosomes were isolated

96 from the serum sample using ExoQuick Exosome Precipitation Solution (System Biosciences,

97 CA, USA). Exosomes were characterized by electron microscopy (FEI Quanta 250, Thermo

98 Fisher Scientific, Eindhoven, the Netherlands), nano particle tracking analysis (NTA) (ZetaView

99 PMX 110, Particle Metrix, Meerbusch, Germany), and Western blot analysis (for CD9, HSP70).

100

$101 \quad 2.3$ Exosomes miRNA sequencing and sequence analysis

102 Samples were collected from 4 PCOS patients and 4 non-PCOS volunteers. The kit and 103 protocol used were the Illumina TruSeq RNA Sample Preparation kit v2 (catalog \# RS-1 104 22-2001) and the TruSeq RNA Sample Preparation v2 Guide (part \# 15026495). The libr 105 aries were sequenced on an Illumina HiSeq X Ten platform. Sequencing was performed 106 by paired - end sequencing $(2 \times 150 \mathrm{bp})$ on Illumina HiSeqX to a minimal depth of 30 $107 \times$ base coverage. Raw sequence alignment and variant calling were carried out using Ill 108 umina CASAVA 1.8 software. To quantify microRNAs, back-spliced junction reads were sca 109 led to RPKM mapped reads.

110

111 miRWalk (http://mirwalk.uni-hd.de) and miRBase 22.0 (http://www.mirbase.org) were used 112 to analyze the miRNA sequences. The FastQC software (http://www.bioinformatics.bbsrc.a $113 \mathrm{c} . u k /$ projects/fastqc/) was used to make sure that the sequencing data were high quality.

114 The heatmap was plotted based on the log2(fold change), using Heatmap Illustrator softw 115 are (Heml 1.0). In addition, gene ontology (GO) and pathway analyses were performed $t$ 116 o identify miRNA-related genes, pathways and GO terms based on sequencing data sets. 117 GO analysis was performed using DAVID (http://david.abcc.ncifcrf.gov/). The RNAhybrid 118 and miRanda database were used to predict potential target genes of all the differentially 119 expressed miRNAs. Set-up parameters were as follows: RNAhybird energy <-25; miRand 120 a energy $<-20$ and score $\geqslant 150$.

\subsection{Western blotting analysis}

123 Western blotting was performed as we previously described ${ }^{9}$, four exosomes of human serum 
124 were prepared from control groups and four samples from PCOS groups, separately. Samples

125 were separated in a 10\% SDS gel. The separated samples were transferred to a nitrocellulose 126 transfer membrane (Bio-Rad, Hercules, CA, USA). After incubating for 1 hour with blocking

127 buffer, the membrane was incubated overnight at $4^{\circ} \mathrm{C}$ with primary antibodies against Hsp70

128 (Beyotime AF1156, Shanghai, China, 1:1,000), CD9 (Beyotime AF1192, Shanghai, China, 129 1:1,000), beta Tubulin (Beyotime AF1216, Shanghai, China, 1:1,000) and Calnexin (Beyotime 130 AC018, Shanghai, China, $1: 1,000)$ at $4^{\circ} \mathrm{C}$ overnight. After three washes with $1 \times \mathrm{TBST}, \mathrm{pH} 7.4$, 131 each membrane was incubated with the appropriate secondary antibody (1:1000) at room 132 temperature for $1 \mathrm{~h}$. After additional three washes, protein intensities were visualized with the 133 enhanced ECL detection system (Sage Creation, Beijing, China). Each experiment was repeated 134 three times.

135

\subsection{RT-qPCR analysis}

RT-qPCR was performed as we previously described ${ }^{9}$. Total miRNAs from the serum exosomes were extracted using the miRcute miRNA isolation kit (TIANGEN, Beijing, China). Real-time PCR was performed for validation using miRcute miRNA qPCR Detection Kit (SYBR Green) (TIANGEN, Beijing, China) after polyadenylation and reverse transcription (miRcute miRNA

141 First-Strand cDNA Synthesis Kit, TIANGEN, China). All miRNA primers were purchased from 142 Tiangen (Beijing, China). The relative microRNA levels were normalized to U6 expression for each sample. The microRNA primers used in the study are presented in Table 2 . The reactions were performed with a StepOne Plus Real-Time PCR System (Applied Biosystems) and StepOne software v2.1. The PCR reaction included a fast start step of $15 \mathrm{~min}$ at $95{ }^{\circ} \mathrm{C}$ followed by 45 cycles of amplification where each cycle consisted of denaturation at $94{ }^{\circ} \mathrm{C}$ for $20 \mathrm{~s}$ and annealing at $60{ }^{\circ} \mathrm{C}$ for $34 \mathrm{~s}$. Melting curve analysis was performed to verify the identity and specificity of the PCR products. Analyses of gene expression was performed by the $2^{-\Delta \Delta \mathrm{Ct}}$ method. Each experiment was repeated three times.

\subsection{Statistical analysis}

152 Data are displayed as the means \pm standard deviations (SD). The statistical significance of the 153 results from three independent assays was evaluated by Student's t-test. $P<0.05$ was considered 154 to indicate statistically significant differences. 
155

\section{Results}

157 3.1 Clinical information of PCOS group and normal group

158 Exosomes were purified from the normal controls $(n=4)$ and PCOS participants' $(n=4)$ serum.

159 PCOS patients had a mean age of $28.1( \pm 1.72)$ years and healthy controls had a mean age of 26.8

$160( \pm 1.27)$ years. There were no significant differences of body mass index (BMI) between the

161 patients and the matched controls. Participant clinical characteristics are shown in Table 1.

162

163

\subsection{Isolation and characterization of serum-derived EXOs}

164

The characteristics and properties of isolated exosomes are described in Figure 1.Western blot

165 analysis validated the expression of the known exosomal biomarkers CD9 and HSP70 (Figure.

1A). The size and morphology feature of exosomes were examined by transmission electron microscopy (scale bar, $200 \mathrm{~nm}$ ) (Figure. 1B). NTA analysis showed that exosomes isolated from serum had a diameter size range of $\sim 154.7 \pm 9 \mathrm{~nm}$ (Figure. 1C), consistent with the known size of exosomes.

170

\subsection{Analysis of miRNAs in serum EXOs}

172

In the present study, a total of 1475 miRNAs were identified in serum exosomes from both PCOS and the controls (Supplemental Table S1). The original sequencing data were uploaded to the NCBI SRA database (https:/www.ncbi.nlm.nih.gov/sra) with the accession number PRJNA646028. Statistical analysis of the exosomal microRNAs sequencing results revealed that the levels of 54 mature miRNAs in serum exosomes from the PCOS group differed significantly

177 from those in control group $\left(P<0.05, \log _{2}|\mathrm{FC}|>1.2\right)$ (Supplemental Table S2). As shown in

178 Figure. 2, hierarchical clustering for the 54 differentially expressed miRNAs, including 27 upregulated miRNAs and 27 downregulated miRNA, is indicated by a heatmap and a volcano 180 plot.

qPCR was performed for miRNAs with significant changes based on the miRNA sequencing data (Figure. 3). We confirmed upregulation of hsa-miR-1299, hsa-miR-6818-5p and downregulation of hsa-miR-192-5p, hsa-miR-145-5p in serum exosomes from women with and without PCOS. In addition, we confirmed that the FANCC is a miR-1299 target gene (Figure S4). 
186

187 3.4 GO and pathway analysis of miRNA target genes

188 It was observed that 54 differentially expressed miRNAs can target 28502 differentially

189 expressed genes (Figure. S1, Supplemental Table S3). Furthermore, Gene Oncology (GO)

190 (Figure. S2) and Kyoto Encyclopedia of Genes and Genomes (KEGG) pathway analysis (Figure.

191 4) was performed to investigate the functions of their target genes. In addition, KEGG pathways

192 enrichment revealed that the DEGs were mainly involved in the axon guidance, pathways in

193 cancer and MAPK signaling pathway.

194

195 Discussion

196 Scrutinizing the role and differential expression of miRNAs in various tissues of PCOS patients

197 has been the subject of increasing number of studies, which generally focused on miRNAs as

198 potential biomarkers of the syndrome ${ }^{10}$. Multiple independent studies have reported that

199 exosomes contain miRNA, and exosomes have been proposed to be treasure chests for biomarker

200 applications $^{11}$. Furthermore, miRNAs have recently emerged as ideal molecules that could be

201 used as diagnostic and prognostic biomarkers in many diseases ${ }^{12}$. In this study, we performed a

202 comprehensive sequence-based characterization of the PCOS serum exosomal miRNAs, the

203 sequence data revealed that the levels of 54 miRNAs significantly differed between PCOS

204 patients and normal controls. As shown in Figure S3, the PCA graph has enabled a good

205 discrimination between the samples. The PCA two-dimensional spatial distribution was

206 consistent with that of the heat map analysis. Our findings in this study demonstrate that hsa-

207 miR-1299, hsa-miR-6818-5p hsa-miR-192-5p, and hsa-miR-145-5p are differentially expressed

208 in PCOS patients serum exosomes and have the potential to be used as biomarkers in the

209 diagnosis of PCOS.

210

211 Recently, protein profile ${ }^{8}$, small RNA sequences ${ }^{4}$ and circRNAs profile ${ }^{7}$ of follicle fluid

212 exosomes have been identified in polycystic ovary syndrome patients ${ }^{7}$. In addition, exosomal

$213 \mathrm{miR}-323-3 \mathrm{p}$ and $\mathrm{miR}-27 \mathrm{a}-5 \mathrm{p}$ also have been proved may play a role in polycystic ovary

214 syndrome (PCOS). Similarly to Che et al. ${ }^{6}$, we identified potential exosomal miRNAs and their

215 gene targets using sequencing and bioinformatics approaches. However, several of these

216 miRNAs are potentially novel biomarkers for PCOS that have not been mentioned in previous

Peer] reviewing PDF | (2020:05:49119:3:0:NEW 11 Jan 2021) 
217 reports. To confirm this, underlying epigenetic mechanisms should be further investigated.

218 Some studies focusing on alteration miRNAs have been published. miR-1299 has recently been 219 reported as potential biological markers for rheumatic heart disease (RHD) development ${ }^{13}$.

220 Gestational Diabetes Mellitus Regulatory Network Identifies hsa-miR-145-5p as Potential

221 Biomarkers ${ }^{14}$. hsa-miR-192-5p has the potential to be an early diagnostic marker for Venous 222 thrombosis $(\mathrm{VT})^{15}$. hsa-miR-4649-5p has the potential to be Amyotrophic Lateral Sclerosis 223 (ALS) diagnosis biomarkers ${ }^{16}$. miR-6782-5p exhibit the highest expression and are 224 candidate circulating biomarkers for metastatic activity of prostate cancer ${ }^{17}$. miR-4306 acts 225 as a tumor suppressor in TNBC and is a potential therapeutic target for TNBC treatment ${ }^{18}$. 226 hsa-miR-31-5p plays an important role in HS formation by inhibiting FIH and regulating the 227 HIF-1alpha pathway ${ }^{19}$. hsa-miR-143-5p robustly down-regulated in Intracranial aneurysm 228 (IA $)^{20}$. Downregulation hsa-miR-214-3p may suppressed human epithelial ovarian cancer (EOC) 229 development ${ }^{21}$. hsa-mir125b-5p can be a prognostic and diagnostic biomarker for for Stage I 230 Lung Adenocarcinoma ${ }^{22}$. hsa-miR-135b-5p regulates the APC gene in both intestinal and diffuse 231 subtypes of Gastric Cancer (GC) ${ }^{23}$. miR-5701 inhibits the proliferation of cervical cancer cells 232 and the expression of its target gene THBS4 $4^{24}$. hsa-miR-3607-3p may represent as promising 233 therapeutic targets against kidney fibrosis ${ }^{25}$. hsa-miR-592 showed correlation with monosomy 3 234 tumors $^{26}$. The significance of the different expressions of miRNA in various diseases remains to 235 be determined. Therefore, exploring several key biomarkers for PCOS diagnosis and treatment 236 will be of great significance.

237

238 miRNA target-based pathway enrichment analysis of PCOS revealed 284 enriched pathways that 239 included MAPK signaling, Hippo signaling pathway and Insulin signaling pathway

240 (Supplementary Table S4); these pathways are known to be associated with PCOS. TRIB1 can 241 activate the MAPK kinase pathway, which is induced in muscle cells in PCOS women ${ }^{27}$. Hippo 242 gene expression fingerprints could potentially be used to more accurately define patients with $243 \mathrm{PCOS}^{28}$. Insulin resistance is considered as part of the pathogenesis of polycystic ovary 244 syndrome (PCOS), and PCOS patients often show hyperinsulinemia ${ }^{29}$. The exact relationship 245 between insulin resistance and Anti-Müllerian hormone (AMH) has not been fully elucidated. 246 Recent research suggests that the diversity of AMH genotypes in the AMH signal pathway may 247 be connected with the susceptibility and phenotype of PCOS with insulin resistance ${ }^{30}$. Women 
248 with PCOS have high AMH; and accordingly AMH has been proposed as a marker of PCOS 249 with high $\mathrm{AFC}^{31}$. AMH levels have the potential to be diagnostic and prognostic modalities in 250 PCOS patients.

251

252 Conclusion

253 Collectively, in our study, the exosomal microRNA expression profiles between PCOS patients 254 and non-PCOS individuals were comparatively and comprehensively analyzed. hsa-miR-1299, 255 hsa-miR-145-5p, hsa-miR-6818-5p and hsa-miR-192-5p may be potential biomarkers for 256 diagnosis and treatment of PCOS. Nevertheless, identification of potential biomarkers of 257 response was limited by the small sample size. Future research is needed to determine a better 258 diagnostic criteria and ways to improve the diagnostic rate in early stages of PCOS.

259

260 Funding

261 This work was supported by the National Natural Science Foundation of China (81701522 and 262 82071729), the Zhejiang Provincial Natural Science Foundation of China (LY19H040002), the 263 Science Technology Department of Zhejiang Province, China (LGF21H040003, 264 LGF21H040004, LGF19H040004, LGD20H040001, LGF18H180015) and the Health 265 Commission of Zhejiang Province, China (2021KY375, 2021KY1154, 2021KY1157, 266 2020KY1001, 2018KY844, 2018KY845, 2018KY848, 2019KY229, 2019KY230, 2019RC296, 267 2019KY717); the Science Technology Department of Shaoxing, China (2020A13032, 268 2020A13034, 2020A13035, 2020A13037, 2018C30039, 2018C30042, 2018C30043, 269 2018C30044, 2018C30048).

\section{References}

2711 Chen B, Xu P, Wang J, and Zhang C. 2019. The role of MiRNA in polycystic ovary $272 \quad$ syndrome (PCOS). Gene 706:91-96. 10.1016/j.gene.2019.04.082

2732 Kalluri R, and LeBleu VS. 2020. The biology, function, and biomedical applications of $274 \quad$ exosomes. Science 367. 10.1126/science.aau6977

2753 LeBleu VS, and Kalluri R. 2020. Exosomes as a Multicomponent Biomarker Platform in $276 \quad$ Cancer. Trends Cancer. 10.1016/j.trecan.2020.03.007

2774 Hu J, Tang T, Zeng Z, Wu J, Tan X, and Yan J. 2020. The expression of small RNAs in 
278

279

280

281

282

283

$284 \quad 6$

285

286

287

288

289

290

2918

292

293

294

2959

296

297

298

$299 \quad 10$

300

301

$302 \quad 11$

303

304

$305 \quad 12$

306

307

$308 \quad 13$

exosomes of follicular fluid altered in human polycystic ovarian syndrome. Peerj 8:e8640. 10.7717 peerj.8640

5 Zhao Y, Tao M, Wei M, Du S, Wang H, and Wang X. 2019a. Mesenchymal stem cells derived exosomal miR-323-3p promotes proliferation and inhibits apoptosis of cumulus cells in polycystic ovary syndrome (PCOS). Artif Cells Nanomed Biotechnol 47:38043813. 10.1080/21691401.2019.1669619

6 Che X, Jian F, Chen C, Liu C, Liu G, and Feng W. 2020. PCOS serum-derived exosomal miR-27a-5p stimulates endometrial cancer cells migration and invasion. Journal of Molecular Endocrinology 64:1-12. 10.1530/JME-19-0159

7 Wang LP, Peng XY, Lv XQ, Liu L, Li XL, He X, Lv F, Pan Y, Wang L, Liu KF, and Zhang XM. 2019. High throughput circRNAs sequencing profile of follicle fluid exosomes of polycystic ovary syndrome patients. Journal of Cellular Physiology. $10.1002 /$ jcp. 28201

Li H, Huang X, Chang X, Yao J, He Q, Shen Z, Ji Y, and Wang K. 2020. S100-A9 protein in exosomes derived from follicular fluid promotes inflammation via activation of NF-kappaB pathway in polycystic ovary syndrome. Journal of Cellular and Molecular Medicine 24:114-125. 10.1111/jcmm.14642

9 Pan HT, Ding HG, Fang M, Yu B, Cheng Y, Tan YJ, Fu QQ, Lu B, Cai HG, Jin X, Xia XQ, and Zhang T. 2018. Proteomics and bioinformatics analysis of altered protein expression in the placental villous tissue from early recurrent miscarriage patients. Placenta 61:1-10. 10.1016/j.placenta.2017.11.001

10 Jiang L, Li W, Wu M, and Cao S. 2015. Ciculating miRNA-21 as a Biomarker Predicts Polycystic Ovary Syndrome (PCOS) in Patients. Clinical Laboratory 61:1009-1015. 10.7754/clin.lab.2015.150122

11 Cheng L, Sharples RA, Scicluna BJ, and Hill AF. 2014. Exosomes provide a protective and enriched source of miRNA for biomarker profiling compared to intracellular and cell-free blood. J Extracell Vesicles 3. 10.3402/jev.v3.23743

2 Zhang Y, Han T, Feng D, Li J, Wu M, Peng X, Wang B, Zhan X, and Fu P. 2019. Screening of non-invasive miRNA biomarker candidates for metastasis of gastric cancer by small RNA sequencing of plasma exosomes. Carcinogenesis. 10.1093/carcin/bgz186 13 Li N, Lian J, Zhao S, Zheng D, Yang X, Huang X, Shi X, Sun L, Zhou Q, Shi H, Xu G, 
309

310

311

$312 \quad 14$

313

314

315

$316 \quad 15$

317

318

$319 \quad 16$

320

321

322

323

324

325

$326 \quad 18$

327

328

329

330

331

332

$333 \quad 20$

334

335

$336 \quad 21$

337

338

$339 \quad 22$

Incoom EK, Zhou J, and Shao G. 2015. Detection of Differentially Expressed MicroRNAs in Rheumatic Heart Disease: miR-1183 and miR-1299 as Potential Diagnostic Biomarkers. Biomed Res Int 2015:524519. 10.1155/2015/524519 Zamanian Azodi M, Rezaei-Tavirani M, Rezaei-Tavirani M, and Robati RM. 2019. Gestational Diabetes Mellitus Regulatory Network Identifies hsa-miR-145-5p and hsamiR-875-5p as Potential Biomarkers. Int J Endocrinol Metab 17:e86640. 10.5812/ijem. 86640

15 Rodriguez-Rius A, Lopez S, Martinez-Perez A, Souto JC, and Soria JM. 2020. Identification of a Plasma MicroRNA Profile Associated With Venous Thrombosis. Arterioscler Thromb Vasc Biol 40:1392-1399. 10.1161/ATVBAHA.120.314092 Takahashi I, Hama Y, Matsushima M, Hirotani M, Kano T, Hohzen H, Yabe I, Utsumi J, and Sasaki H. 2015. Identification of plasma microRNAs as a biomarker of sporadic Amyotrophic Lateral Sclerosis. Mol Brain 8:67. 10.1186/s13041-015-0161-7

17 Fomicheva KA, Osip'yants AI, Knyazev EN, Samatov TR, and Shkurnikov MY. 2017. Detection of Potential Metastatic Prostate Cancer Circulating Biomarkers by Comparison of miRNA Profiles in DU145 Cells and Culture Medium. Bull Exp Biol Med 162:792796. 10.1007/s10517-017-3715-0

18 Zhao Z, Li L, Du P, Ma L, Zhang W, Zheng L, Lan B, Zhang B, Ma F, Xu B, Zhan Q, and Song Y. 2019b. Transcriptional Downregulation of miR-4306 serves as a New Therapeutic Target for Triple Negative Breast Cancer. Theranostics 9:1401-1416. 10.7150/thno. 30701

19 Wang X, Zhang Y, Jiang BH, Zhang Q, Zhou RP, Zhang L, and Wang C. 2017. Study on the role of Hsa-miR-31-5p in hypertrophic scar formation and the mechanism.

Experimental Cell Research 361:201-209. 10.1016/j.yexcr.2017.09.009

20 Jiang Y, Zhang M, He H, Chen J, Zeng H, Li J, and Duan R. 2013. MicroRNA/mRNA profiling and regulatory network of intracranial aneurysm. BMC Med Genomics 6:36. 10.1186/1755-8794-6-36

21 Wang C, Qi S, Xie C, Li C, Wang P, and Liu D. 2018. Upregulation of long non-coding RNA XIST has anticancer effects on epithelial ovarian cancer cells through inverse downregulation of hsa-miR-214-3p. J Gynecol Oncol 29:e99. 10.3802/jgo.2018.29.e99 Zeybek A, Oz N, Kalemci S, Edgunlu T, Kiziltug MT, Tosun K, Tunc M, Tekin L, and 
340

341

342

343

344

345

346

347

$348 \quad 24$

349

350

351

352

353

354

355

356

357

358

359

360

361

362

$363 \quad 28$

364

365

366

367

368

Erdal ME. 2019. Diagnostic Value of MiR-125b as a Potential Biomarker for Stage I Lung Adenocarcinoma. Current Molecular Medicine 19:216-227. $10.2174 / 1566524019666190314113800$

23 Magalhaes L, Quintana LG, Lopes DCF, Vidal AF, Pereira AL, D'Araujo Pinto LC, de Jesus Viana Pinheiro J, Khayat AS, Goulart LR, Burbano R, de Assumpcao PP, and Ribeiro-Dos-Santos A. 2018. APC gene is modulated by hsa-miR-135b-5p in both diffuse and intestinal gastric cancer subtypes. Bmc Cancer 18:1055. 10.1186/s12885-0184980-7

24 Prajapati P, Sripada L, Singh K, Roy M, Bhatelia K, Dalwadi P, and Singh R. 2018. Systemic Analysis of miRNAs in PD Stress Condition: miR-5701 Modulates Mitochondrial-Lysosomal Cross Talk to Regulate Neuronal Death. Mol Neurobiol 55:4689-4701. 10.1007/s12035-017-0664-6

25 Yu J, Yu C, Feng B, Zhan X, Luo N, Yu X, and Zhou Q. 2019. Intrarenal microRNA signature related to the fibrosis process in chronic kidney disease: identification and functional validation of key miRNAs. BMC Nephrol 20:336. 10.1186/s12882-019-1512$\mathrm{X}$

26 Wroblewska JP, Lach MS, Ustaszewski A, Kulcenty K, Ibbs M, Jagiello I, Suchorska WM, and Marszalek A. 2020. The Potential Role of Selected miRNA in Uveal Melanoma Primary Tumors as Early Biomarkers of Disease Progression. Genes (Basel) 11. $10.3390 /$ genes 11030271

27 Liu YH, Tan KA, Morrison IW, Lamb JR, and Argyle DJ. 2013. Macrophage migration is controlled by Tribbles 1 through the interaction between C/EBPbeta and TNF-alpha. Vet Immunol Immunopathol 155:67-75. 10.1016/j.vetimm.2013.06.001

28 Maas K, Mirabal S, Penzias A, Sweetnam PM, Eggan KC, and Sakkas D. 2018. Hippo signaling in the ovary and polycystic ovarian syndrome. J Assist Reprod Genet 35:17631771. $10.1007 / \mathrm{s} 10815-018-1235-0$

29 Zhang C, Yu C, Lin Z, Pan H, Li K, and Ma H. 2020. MiRNAs expression profiling of rat ovaries displaying PCOS with insulin resistance. Archives of Gynecology and Obstetrics 302:1205-1213. 10.1007/s00404-020-05730-z 
36930 Sahmay S, Aydogan Mathyk B, Sofiyeva N, Atakul N, Azemi A, and Erel T. 2018. 370 Serum AMH levels and insulin resistance in women with PCOS. Eur J Obstet Gynecol $371 \quad$ Reprod Biol 224:159-164. 10.1016/j.ejogrb.2018.03.007

37231 Gupta M, Yadav R, Mahey R, Agrawal A, Upadhyay A, Malhotra N, and Bhatla N. 2019. 373 Correlation of body mass index (BMI), anti-mullerian hormone (AMH), and insulin resistance 374 among different polycystic ovary syndrome (PCOS) phenotypes - a cross-sectional study.

375 Gynecological Endocrinology 35:970-973. 10.1080/09513590.2019.1613640 


\section{Figure Legends}

377 Figure 1. Characterization of exosomes derived from human serum.

378 (A) Western blot analysis of the surface markers of exosomes (Hsp70 and CD9).

379 (B) Serum-derived exosomes visualized by electron microscopy (scale bar, $200 \mathrm{~nm}$ ). (C) The

380 representative nanoparticle tracking analysis (NTA) profile of exosomes from human serum.

381

382 Figure 2. Cluster analysis of differentially expressed exosomal miRNAs isolated from serum 383 from PCOS patients and normal controls.

384 (A) Heat map with hierarchical clustering (HCL) of normalized microRNAs abundance reveals 385 the 54 differentially expressed microRNAs from PCOS $(n=4)$ and normal control $(n=4)$.

386 (B) Volcano map showing the distribution of differential microRNAs according to their $\mathrm{p}$ values 387 and fold-changes. Candidates with $\mathrm{p}<0.05$ and $\mid \log 2$ (fold-change) $\mid \geq 1$ are considered 388 differential.

389 (C) The top five most up-and downregulated microRNAs.

390

391 Figure 3. Validation of selected miRNAs by qRT-PCR.

$392 * p<0.05$; miRNA: micro RNA; PCOS: polycystic ovary syndrome; qRT-PCR: quantitative 393 reverse-transcription polymerase chain reaction

394

395

Figure 4. KEGG pathway analysis for the predicted target genes of the differentially expressed miRNAs.

397 (A) Coordinate axis X:- $\log _{10}(P$-value) ; coordinate axis Y: Pathway-Term entry name. (B)

398 Coordinate axis $\mathrm{X}:-\log _{10}(P$-Value $)$; coordinate .axis Y: Pathway-Term entry name.

399

400 Figure S1. Venn chart of the target genes of differentially expressed miRNAs

401 RNAhybrid : Energy $<-25$

402 Miranda : score $>150$ 、Energy $<-20$

403

404 Figure S2. Gene ontology (GO) enrichment analysis of predicted target genes.

405 (A) GO analysis of all the differentially expressed genes included: Biological Process (BP), 406 Molecular Function (MF), and Cellular Component (CC). 
407 (B) Bubble plot of representative GO enrichment results of differentially expressed genes.

408 Figure S3. Principal component analysis

409 Figure S4. Validation of the predicted miR-1299 target gene.

410 Figure S5. Speculative schematic model 
Table $\mathbf{1}$ (on next page)

Table_1

Clinical information of PCOS group and normal group 


\begin{tabular}{|c|c|c|c|c|}
\hline Parameter & $\begin{array}{l}\text { Reference } \\
\text { range }\end{array}$ & $\begin{array}{l}\text { PCOS group } \\
(n=4)\end{array}$ & $\begin{array}{l}\text { Normal } \\
\operatorname{group}(n=4)\end{array}$ & $P$-value \\
\hline Age (years) & & $28.5 \pm 1.73$ & $27.50 \pm 1.29$ & 0.390 \\
\hline BMI (kg/m2) & & $20.85 \pm 1.35$ & $20.45 \pm 0.62$ & 0.610 \\
\hline FSH (IU/L) & $3.3-7.9$ & $7.92 \pm 0.74$ & $5.56 \pm 0.32$ & 0.001 \\
\hline LH (IU/L) & $2.0-12.0$ & $16.38 \pm 2.00$ & $8.55 \pm 1.85$ & 0.001 \\
\hline AMH (ng/ml) & $3.31-7.98$ & $11.30 \pm 1.22$ & $6.21 \pm 1.31$ & 0.001 \\
\hline PRL (ng/ml) & $3.5-24.2$ & $16.23 \pm 4.72$ & $15.33 \pm 3.18$ & 0.742 \\
\hline E2 (pmol/L) & $18-195$ & $64.11 \pm 13.24$ & $53.83 \pm 10.60$ & 0.271 \\
\hline Progesterone (ng/mL) & $0.2-1.2$ & $0.72 \pm 0.15$ & $0.52 \pm 0.28$ & 0.259 \\
\hline Testosterone (ng/mL) & $0.15-0.51$ & $0.71 \pm 0.20$ & $0.38 \pm 0.09$ & 0.022 \\
\hline
\end{tabular}

2 Note. AMH: anti-mullerian hormone; BMI: body mass index; FSH: follicle-stimulating hormone;

3 LH: follicle-stimulating hormone; PCOS: polycystic ovary syndrome; PRL: prolactin. 
Table 2 (on next page)

Table_2

The microRNA primers used in the study 
Table 2. The microRNA primers used in the study

\begin{tabular}{|c|c|}
\hline microRNA & primers \\
\hline hsa-miR-1299 & $\begin{array}{c}5{ }^{\prime}- \\
\text { UUCUGGAAUUCUGUGUGAGGGA- } \\
3 \text {, }\end{array}$ \\
\hline hsa-miR-6818-5p & $\begin{array}{c}5^{\prime}- \\
\text { UUGUGUGAGUACAGAGAGCAUC- } \\
3 \text { ' }\end{array}$ \\
\hline hsa-miR-6818-5p & $\begin{array}{c}\text { 5'-CUGACCUAUGAAUUGACAGCC- } \\
3 \text { ' }\end{array}$ \\
\hline hsa-miR-145-5p & $\begin{array}{c}5^{\prime}- \\
\text { GUCCAGUUUUCCCAGGAAUCCCU- } \\
3{ }^{\prime}\end{array}$ \\
\hline
\end{tabular}

2 
Figure 1

Figure 1. Characterization of exosomes derived from human serum.

(A) Western blot analysis of the surface markers of exosomes (Hsp70 and CD9). (B) Serumderived exosomes visualized by electron microscopy (scale bar, $200 \mathrm{~nm}$ ). (C) The representative nanoparticle tracking analysis (NTA) profile of exosomes from human serum.

A
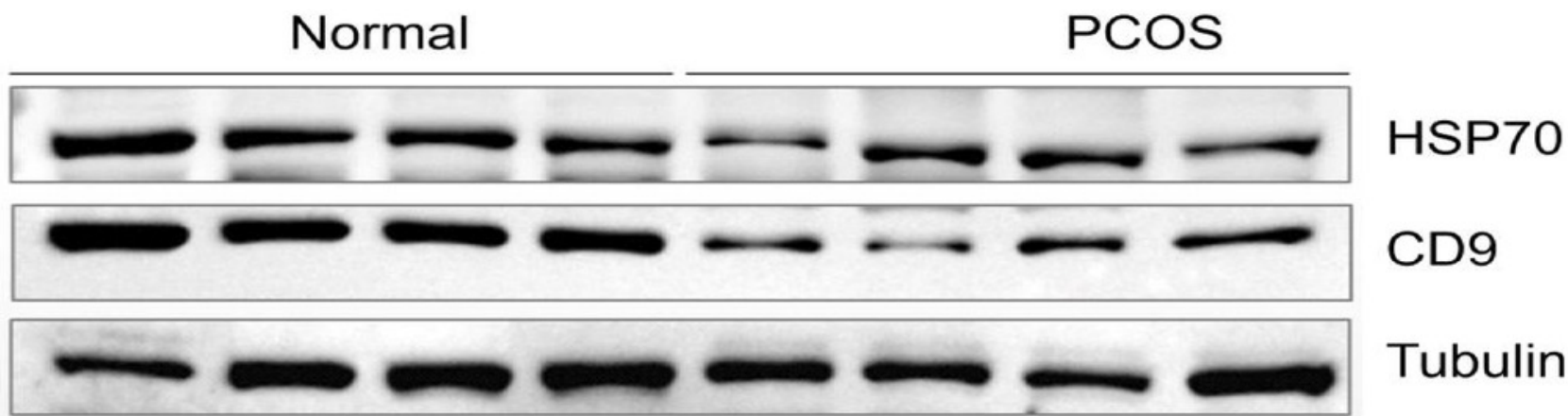

Tubulin

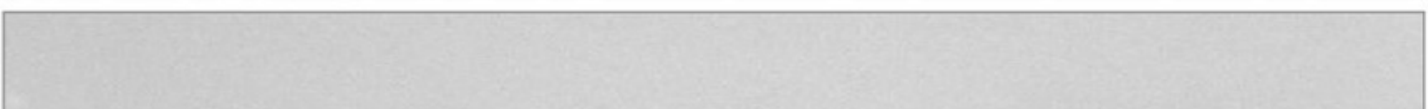

Calnexin

B
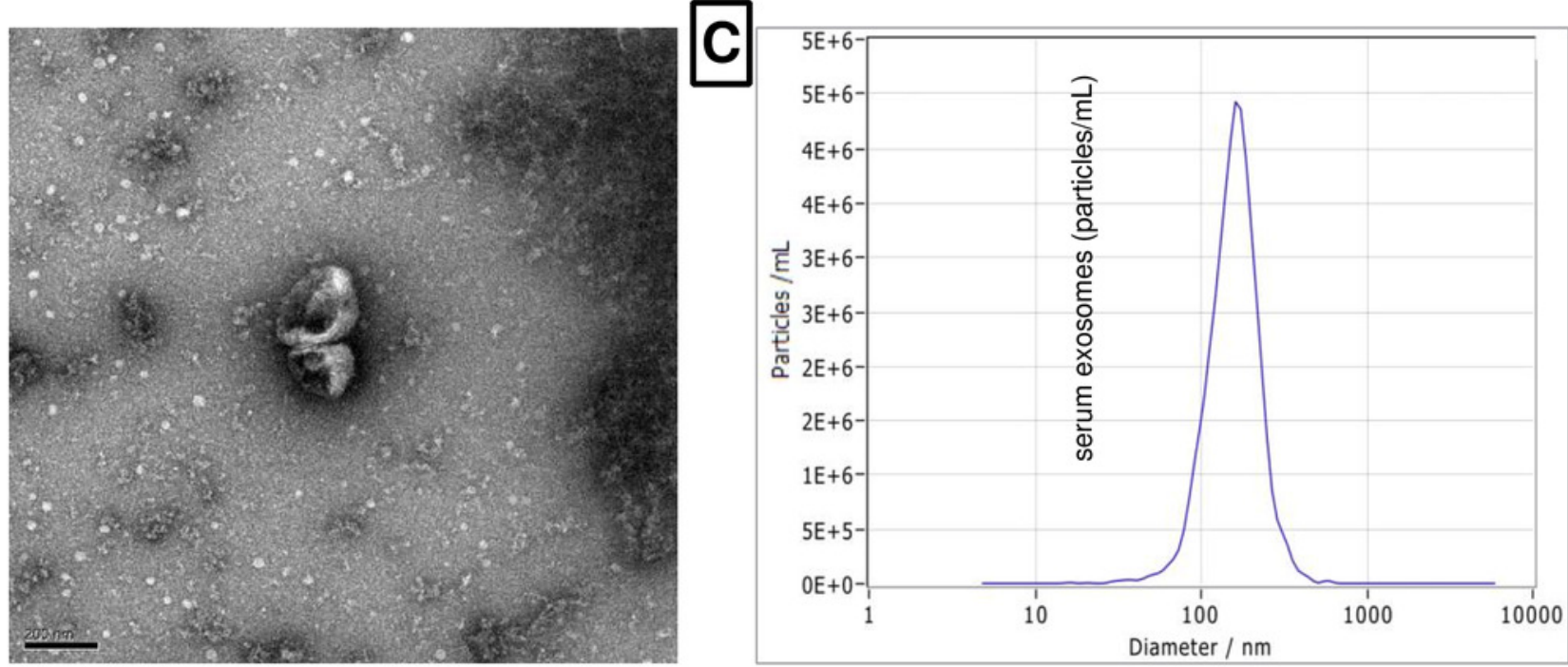
Figure 2

Figure 2. Cluster analysis of differentially expressed exosomal miRNAs isolated from serum from PCOS patients and normal controls.

(A) Heat map with hierarchical clustering $(\mathrm{HCL})$ of normalized microRNAs abundance reveals the 54 differentially expressed microRNAs from PCOS $(n=4)$ and normal control $(n=4)$. (B) Volcano map showing the distribution of differential microRNAs according to their $p$ values and fold-changes. Candidates with $p<0.05$ and $\mid \log 2$ (fold-change) $\mid \geq 1$ are considered differential. (C) The top five most up-and downregulated microRNAs.

A

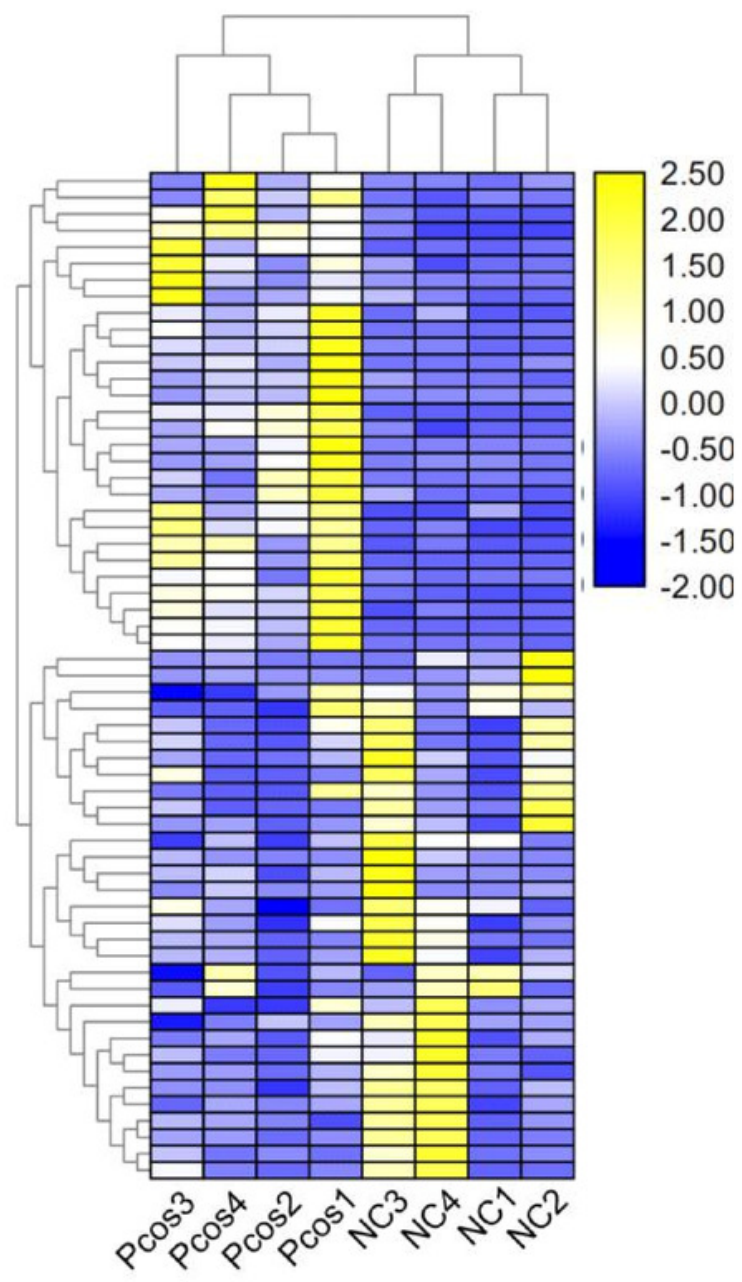

B

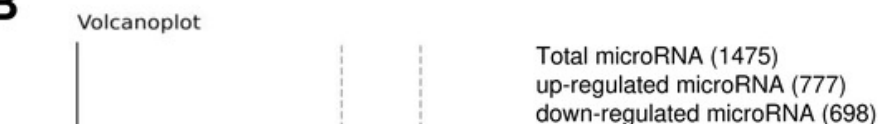

down-regulated microRNA (698)

C

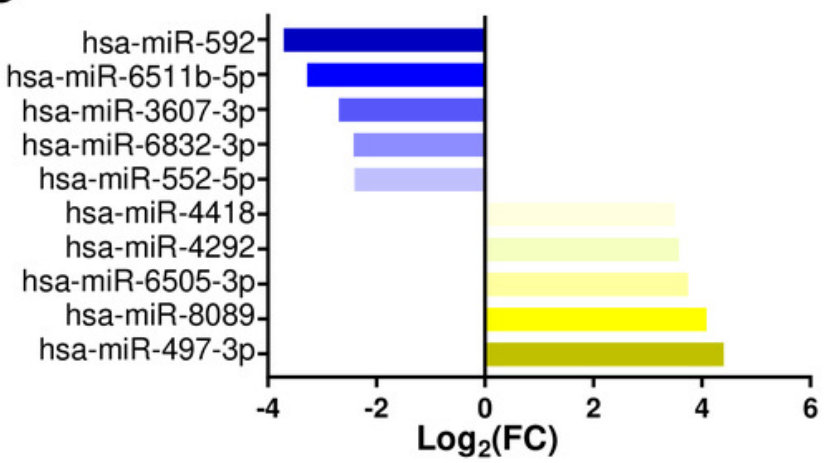


Figure 3

Figure 3. Validation of selected miRNAs by qRT-PCR.

${ }^{*} p<0.05$; miRNA: micro RNA; PCOS: polycystic ovary syndrome; qRT-PCR: quantitative reverse-transcription polymerase chain reaction

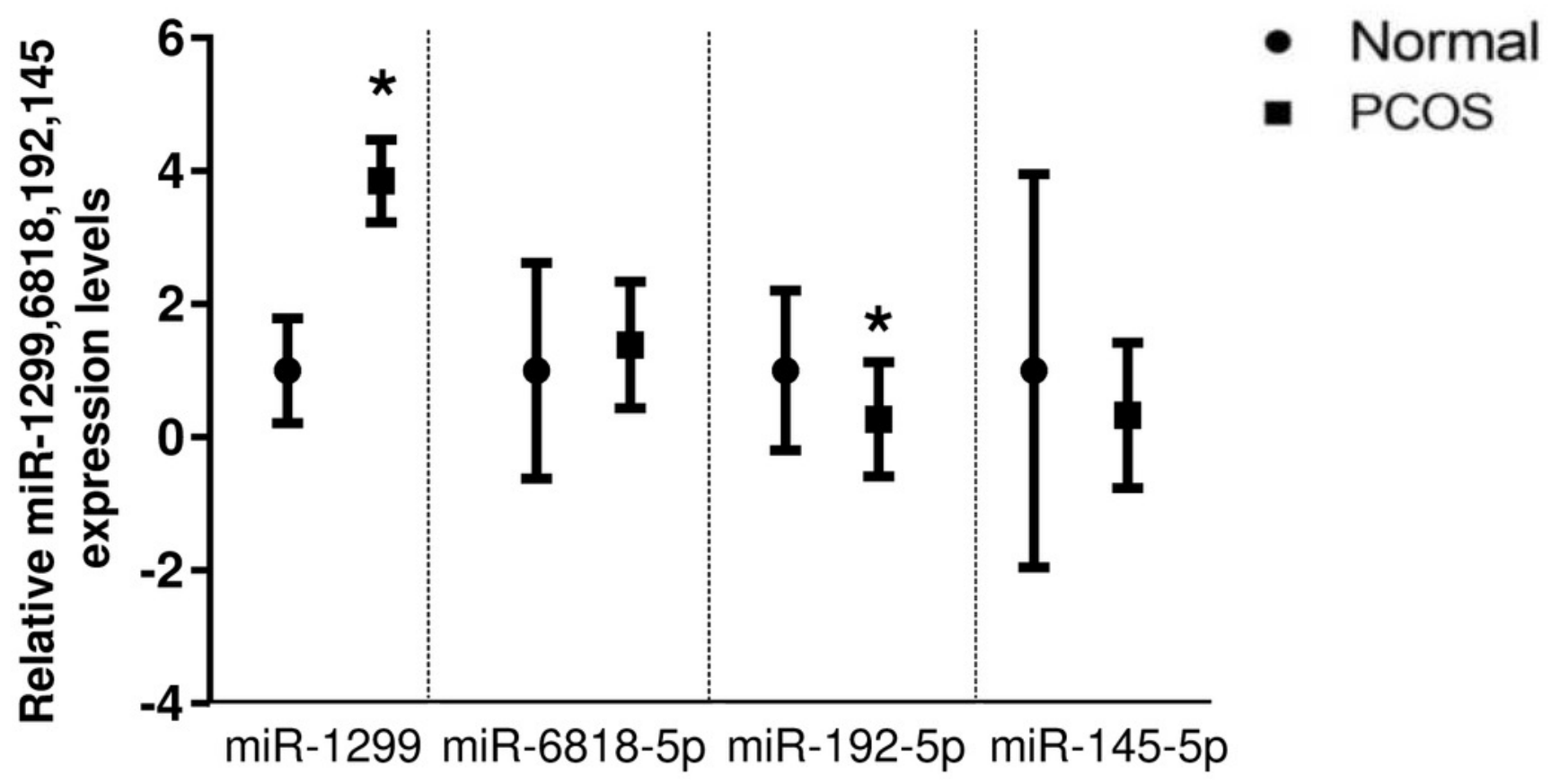


Figure 4

KEGG pathway analysis for the predicted target genes of the differentially expressed miRNAs.

(A) Coordinate axis X:- $\log _{10}(P$-value) ; coordinate axis Y: Pathway-Term entry name. (B)

Coordinate axis X: - $\log _{10}(P$-Value); coordinate .axis Y: Pathway-Term entry name. 
A

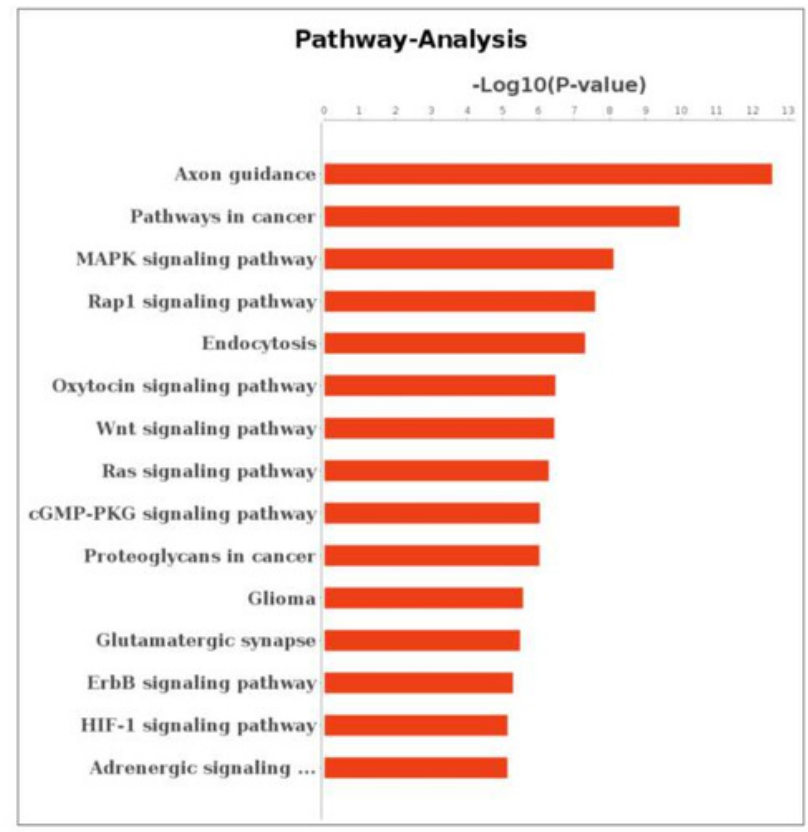

B

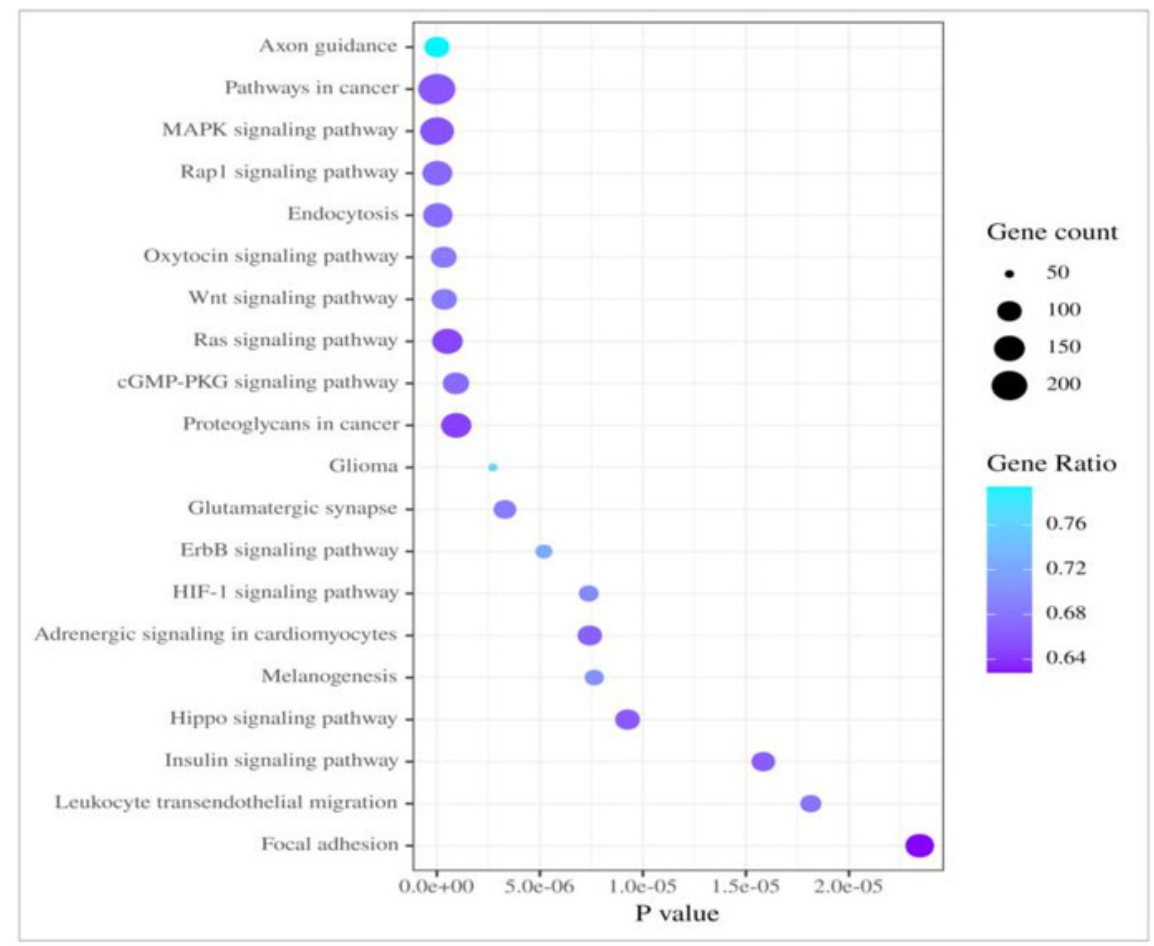

